\title{
El personal de las Compañías Militares y de Seguridad Privadas (CMSP): ¿Contratistas civiles o mercenarios?*
}

The Personnel of the Private Military and Security Companies (PMSC): Civilian Contractors or Mercenaries?

\author{
Mario Iván Urueña-Sánchez ${ }^{\mathrm{a}}$ \\ Universidad del Rosario, Colombia \\ mario.uruena@urosario.edu.co \\ ORCID: https://orcid.org/0000-0002-8040-6240
}

DOI: https://doi.org/10.11144/Javeriana.vj69.epcm

Recibido: 16 Septiembre 2019

Aceptado: 04 Octubre 2019

Publicado: 21 Diciembre 2020

\section{Resumen:}

Este artículo de investigación tiene como propósito explorar la viabilidad y las ventajas derivadas de vincular el estatuto jurídico del personal de las Compañías Militares y de Seguridad Privada con otras figuras jurídicas más desarrolladas para ayudar a dinamizar el derecho internacional. Para tal efecto, se realiza una revisión documental basada en tratados internacionales, jurisprudencia y legislación interna de países emblemáticos. El artículo concluye que el encuadramiento jurídico del personal de estas compañías como mercenarios puede ayudar de algún modo con los problemas de tipificación, aplicabilidad y fuerza vinculante que enfrentan las normas pertinentes.

Palabras clave: CMSP, mercenarismo, contratistas privados, derecho internacional humanitario, derechos humanos.

\begin{abstract}
:
This research article aims to explore the viability and advantages of linking the legal status of the personnel of the Private Military and Security Companies with other more developed legal figures to help boost international law. Thus, a documentary review based on international treaties and domestic jurisprudence and legislation of emblematic countries is carried out. The article concludes that the legal classification of the personnel of these companies as mercenaries can help in some way with the problems of classification, applicability and binding force faced by the relevant regulations.
\end{abstract}

Keywords: PMSC, mercenarism, private contractors, international humanitarian law, human rights.

\section{Introducción}

¿Qué elementos determinan el estatuto jurídico de las Compañías Militares y de Seguridad Privada? ¿Es el personal de estas compañías civil o combatiente? ¿Pueden ser ellos considerados como mercenarios? El vertiginoso aumento en la densidad y profundidad en las relaciones entre unidades del sistema internacional conlleva a la emergencia de nuevos actores que colman espacios en la satisfacción de nuevas necesidades globales. Una de esas necesidades cuya demanda se elevó exponencialmente es la seguridad. El surgimiento de nuevas y diferentes amenazas ha superado la capacidad que tienen los Estados para cumplir con su objetivo de monopolizar la violencia. Sobre esa base, las empresas transnacionales destinadas a prestar el servicio militar de seguridad se multiplicaron y diversificaron su portafolio. En el argot internacional, estas empresas han tomado el nombre (no sin disenso) de Compañías Militares y de Seguridad Privadas (en adelante CMSP).

Tales CMSP han logrado abarcar funciones que antes eran exclusivas de los Estados nación, lo que les ha hecho contraer simpatizantes y detractores. Más importante aún, esta circunstancia ha puesto a prueba la capacidad de reacción y de dinamismo que debería caracterizar al derecho internacional ante los nuevos desafíos de la era actual. Infortunadamente, el derecho internacional se ha quedado corto frente a la situación al arriesgarse a convertirse en cómplice de injusticias a nivel mundial. Es imperativo entonces examinar el problema más allá de la óptica jurídica tradicional.

Notas de autor

\footnotetext{
${ }^{a}$ Autor de correspondencia. Correo electrónico: mario.uruena@urosario.edu.co
} 
Por ende, la idea central de este artículo defiende que la debilidad del derecho internacional para dar herramientas de regulación a los contratistas de las CMSP o para vincularlos a otras figuras jurídicas mejor tipificadas refleja un problema eminentemente político que alimenta un estatus quo antidemocrático.

Para dar fondo a esta idea, se plantea una metodología fundada en el análisis de textos jurídicos como tratados internacionales, borradores de convenciones, jurisprudencia de cortes nacionales, legislación doméstica e informes gubernamentales de países cuyo sistema normativo sea más desarrollado con respecto al presente tema. Además, se recurrirá a literatura académica que brinda profundidad teórica y conceptual al debate jurídico propuesto.

Este artículo se divide en dos partes. En la primera parte, se establecerá una discusión jurídica que da cuenta de la reglamentación universal, regional y nacional vigente sobre el asunto de las CSMP y sus contratistas. En la segunda parte, se explorarán figuras jurídicas alternativas con las que se pueda realizar una categorización más apropiada de los contratistas militares privados en el derecho internacional.

\section{El debate jurídico en torno a las CMSP}

La realidad política e internacional de las CMSP y su vertiginoso auge guardan poca relación con su realidad jurídica. Un análisis multinivel del marco de regulación de estas empresas conduce a aseverar que i) si bien existe un marco jurídico que pretende regular las CMSP, éste parece diluirse en la medida en que el nivel de análisis se universaliza, ii) el marco jurídico relacionado con las CMSP tiene serios problemas con su tipificación, fuerza vinculante y su aplicabilidad, iii) se debe contemplar la opción de considerar otras figuras jurídicas más desarrolladas en el derecho internacional.

\section{El análisis de fuentes}

En la medida en que el derecho internacional ha ido evolucionando desde su origen hasta nuestros días, las visiones Estado-céntricas que dominaron este campo de estudio han dado paso a la inclusión de nuevos sujetos y actores. La adquisición de subjetividad por parte del individuo y de las Organizaciones Intergubernamentales (OIG) atestiguan tal situación.

Por otra parte, junto a las Organizaciones no Gubernamentales (ONG), las Empresas Transnacionales se han labrado un camino importante al momento de dinamizar el derecho internacional para incluir nuevos temas en la agenda internacional y densificar los lazos de dependencia entre diferentes instancias. No obstante, no todas las empresas mencionadas se dedican a lo mismo y hay un grupo de ellas que, a pesar de moverse en el ámbito de lo privado, su accionar puede terminar teniendo una fuerte repercusión en el derecho público. Un ejemplo evidente de ello es el de las CMSP. La irrupción de estas compañías en el mercado de la seguridad ha facilitado la integridad y el cumplimiento de funciones de las ONG y las OIG, así como ha ayudado a preservar infraestructura estratégica y ha salvaguardado la vida de cierto personal. Sin embargo, el actuar de muchas de estas compañías ha distado de estar exento de polémica, particularmente en áreas que son tan sensibles al derecho internacional público como lo son los derechos humanos (DDHH) y el derecho internacional humanitario (DIH). Es tal la polémica que los debates jurídicos sobre la participación de las CMSP en zonas del mundo que se caracterizan por su fragilidad institucional y humanitaria han terminado por involucrar a los mismos principios generales del derecho internacional.

Causa fuerte curiosidad el que la especial necesidad de regulación de estas compañías sea inversamente proporcional a la existencia de un marco jurídico sólido que universalice estándares para el comportamiento de las CMSP y de su personal. Tal vez el único texto que cumpla este requisito desde lo humanitario es el Documento de Montreux ${ }^{1}$, el cual es una herramienta de soft law que pretende fijar responsabilidades hacia dos partes: hacia los Estados de manera explícita, y hacia las propias compañías por la vía de la sugerencia ${ }^{2}$. A 
los primeros, Montreux los exhorta, por un lado, para que no contraten a este tipo de empresas para actividades que el derecho internacional humanitario reserva a agentes o autoridades estatales y, por el otro, para que garanticen el cumplimiento de las normas y no colaboren en la infracción de este derecho. ${ }^{3}$

El tema con las segundas se relativiza tanto por la obligatoriedad de lo que en el documento se consigna como por el estatuto de su personal. De una parte, la obligatoriedad para hacer que las CMSP respeten el DIH descansa prioritariamente en los Estados, lo que debilita la posibilidad de hacer de las CMSP sujeto de regulación internacional. De la otra parte, pese a que Montreux otorga inicialmente el estatuto de civil al personal de las compañías, éste puede cambiar si él se incorpora a las fuerzas armadas del Estado contratante o si el grupo al que pertenece está bajo la responsabilidad de este último. Por lo tanto, este estatuto se relativiza cuando Montreux establece que está determinado caso por y caso y depende del contrato y del tipo de función prestado ${ }^{4}$.

La iniciativa de Montreux ha tenido un mediano éxito en términos de concurso de sujetos del derecho internacional, ya que en 2013 se realizó la conferencia de Montreux +5 , la cual se desarrolló con el apoyo de 49 Estados y tres organizaciones internacionales. La idea común entre el documento y la conferencia es que se pueda ayudar a clarificar las obligaciones y colaborar para hacer transferencia normativa que se cristalice en leyes o políticas públicas de los Estados 5 .

A pesar del limitado alcance de Montreux para reglamentar las CMSP, su espíritu es asumido por una herramienta del derecho privado: El Código Internacional de Conducta para los Proveedores del Servicio de Seguridad Privada de 2010. Al igual que Montreux, este código de conducta hace parte de la Iniciativa Suiza. Un consorcio entre el gobierno suizo y el Comité Internacional de la Cruz Roja (CRICR) para sacar adelante instrumentos jurídicos no vinculantes destinados a Estados y CMSP. En particular, el código reitera el llamado a contar con reglas apropiadas del derecho internacional para regular a las compañías privadas de seguridad ${ }^{6}$.

Este Código de Conducta fija principios específicos en dos ámbitos: la conducta del personal y la administración y el buen gobierno de las compañías. Con respecto al primer ámbito, los ejes centrales hacen alusión al uso de la fuerza, en tanto este se justifique en los principios de autodefensa o defensa de otros (siempre en el marco de la ley nacional): detención, aprehensión de personas, la prohibición a la tortura o a otros tratos crueles e inhumanos, a la explotación sexual y a la violencia de género, al tráfico humano, esclavitud, al trabajo infantil, etc. Con respecto al segundo ámbito, se hace un llamado a incorporar el código en las políticas de la compañía, a ser estrictos en la selección y el veto de su personal, a darles un entrenamiento adecuado y al manejo y entrenamiento en el uso de armas ${ }^{7}$.

Tomando en cuenta que el documento de Montreux y el Código de Conducta son complementarios por cuanto cada uno concentra sus esfuerzos en un destinatario específico (Estados y compañías respectivamente), ninguna de las dos iniciativas va más allá de encuadrarlos en la normatividad existente. Es decir, ambos instrumentos se limitan a recordar a sus destinatarios cuáles son sus obligaciones respecto al sistema internacional de los DDHH, al DIH y a las buenas prácticas corporativas sin establecer nuevos compromisos. Por ende, cabe decir que en el nivel universal el marco jurídico competente para las CMSP carece de originalidad y especialización.

Ante la inexistencia de una reglamentación particular en la esfera regional, esta parte del análisis proseguirá con las normas domésticas relativas a las CMSP. De esta revisión se pueden extraer dos conclusiones: i) que el interés para regular a las CMSP se encuentra más en los países oferentes del servicio de seguridad por intermedio de sus CMSP, que en aquellos en donde éstas operan y ii) que en el ámbito doméstico hay una regulación más dinámica y concordante con el comportamiento de las CMSP.

Los dos casos más emblemáticos sobre reglamentación interna son el de Sudáfrica y el de los Estados Unidos, ambos sede de numerosas CMSP. Del lado sudafricano se encuentra The South African Regulation of Foreign Military Assistance Act de 1998, el cual se funda en el requerimiento de que todas las compañías de seguridad privada con base en ese país deban recibir autorización del gobierno antes de vender sus servicios 
militares en el exterior ${ }^{8}$. Esta norma obligaba, por ejemplo, a que CMSP sudafricanas como Meteoric Tactical Solutions. Erinys contratadas para participar en la intervención estadounidense a Irak en 2003 tuvieran que someterse al visto bueno de su gobierno y del Comité Nacional de Control de Armas Convencionales. No obstante, la ley sudafricana tuvo un alcance insignificante debido a las quejas permanentes de la industria privada sobre las cuales el gobierno hizo caso omiso, creando un ambiente de confianza entre el Estado y las compañías que terminó por torpedear la implementación de la ley?.

Del lado de los Estados Unidos, si bien no hay un marco legal que explícitamente involucre a las CMSP, sí existe legislación pertinente para regular hechos ilícitos que su personal pueda cometer. ${ }^{10}$ No obstante, por la vía de la jurisprudencia se destaca un gran desarrollo jurídico para entender las bases de la regulación de las CMSP desde dos vertientes. En primer lugar, dos casos derivados del Alien Tort Statute en el que iraquíes detenidos por contratistas alegaron tortura, abusos y violencia sexual: el caso Al-Quraishi vs. Nakla y el caso Al Shimari vs. $C A C I^{11}$.

En segundo lugar, se observan dos fallos judiciales en los que se establece la responsabilidad estatal al trabajar de manera conjunta con las CMSP o al apoyar acciones de éstas. En el primer escenario, se halla el caso Arias vs. Dyncorp en el que el tribunal de Washington juzgó el apoyo de la acción estatal con las CMSP por medio de la prueba de acciones conjuntas para concluir que hubo colaboración entre contratistas del Departamento de Estado con los gobiernos de Estados Unidos y Colombia para verter sustancias tóxicas que afectaron a campesinos ecuatorianos. En el segundo escenario está el caso Blum vs. Yaretsky en el que, al plantear que las acciones de las CMSP están apoyadas de manera significativa por el Estado, en últimas terminan siendo acciones de este último, lo cual demuestra que es posible entablar responsabilidad al Estado por hechos ilícitos que particulares o empresas ejecutan con su avenencia o complicidad ${ }^{12}$. Si bien en esas demandas realizadas a las CMSP se entreven argumentos jurídicos interesantes para sancionar el comportamiento de estos agentes privados, todas estas han sido desestimadas por los tribunales competentes o se encuentran en etapa de apelación luego de un fallo favorable a las compañías.

Continuando con el derecho interno, hay otros dos Estados que, aunque contribuyen con un aporte a la discusión de la regulación de las CMSP, reflejan ser excepciones por diferentes motivos. Éstos son Irak y el Reino Unido. El primero es interesante en el sentido en que la instancia encargada de legislar al respecto era una institución poco convencional manejada por otro Estado. La Coalition Provisional Authority administró el país entre 2003 y 2004 y proveyó un conjunto de reglas para las CMSP que operaban allí para que fueran adoptadas por la ley iraquí. Tal vez el referente más importante en este aspecto es la CPA No. 17, en el que las CMSP que violaran estas reglas podían perder la licencia de funcionamiento, algún depósito, pero no ser sancionadas penalmente, al menos no en suelo iraquí ${ }^{13}$. Cabe señalar que la inmunidad otorgada a las CMSP por el CPA No. 17 terminó en el año 2008 con el Status of Force Agreement (SOFA), donde se reconoce la autonomía de Irak frente a las $\mathrm{CMSP}^{14}$.

El Reino Unido se presenta como la otra excepción, ya que, pese a contar con uno de los documentos más pertinentes para la regulación de las CMSP, conocido como el Green Paper (HC 577 de 2002), éste es más un informe sin fuerza de ley y sin decisiones políticas derivadas. Este documento le ofrece al gobierno británico seis opciones de regulación de las CMSP (incluida la opción de no hacer nada, aunque no sea ésta la opción recomendada), sin que éste haya expresado preferencia por alguna de ellas ${ }^{15}$. La voluntad por sacar adelante una legislación en este país para regular a las CMSP fue disminuyendo en la medida en que el cabildeo de los representantes de esta industria afectó el ambiente en el parlamento.

Después de esta comparación entre los planos universal del derecho internacional y el de los derechos internos, se puede asegurar que la regulación disponible para tratar el tema de las CMSP descansa, en últimas, sobre el segundo plano. Lo anterior porque la reglamentación universal disponible termina regresándole la responsabilidad a los Estados. Por la parte tanto el documento de Montreux como la conferencia de Montreux +5 no se tienen las herramientas para vincular a estas compañías debido a las ramas del derecho desde las que 
se trabaja (DIH y DDHH). El DIH sólo es vinculante para las personas y las partes en un conflicto, no para las empresas y el DDHH sólo vincula a los Estados. ${ }^{16}$ Por lo tanto, lo que ambos Montreux establecen es que como tanto el DIH como los DDHH pueden estar integrados en las legislaciones nacionales y pueden ser aplicados a las CMSP, estas compañías estarían obligadas a respetarlos. Es decir, Montreux y Montreux +5 se limitan a clarificar obligaciones y colaborar para que los Estados trasladen reglas internacionales ya existentes en leyes o políticas nacionales ${ }^{17}$.

De su parte, el código de conducta tampoco se presenta como una gran alternativa por más que sus destinatarias sean las empresas proveedoras de servicios de seguridad, ya que su propósito es el de organizar un conjunto de principios comúnmente acordados para las CMSP y volverlos estándar de buen gobierno ${ }^{18}$.

La preocupación sobre la regulación del comportamiento de las CMSP y su personal se acrecienta cuando se observa que ninguno de los países cuya población ha padecido los desmanes de estos actores cuenta con una legislación doméstica apropiada para crear obligaciones a estas compañías. En el caso de Irak, el modelo de regulación fue impuesto por una potencia intervencionista más con el fin de eximir al personal de las CMSP de responsabilidad penal que de garantizar derechos a poblaciones vulnerables. Así las cosas, el control y juzgamiento de actores cuyas tácticas son una amenaza latente para el DIH y los DDHH reside al final en la buena fe de las burocracias de potencias regionales y mundiales.

\section{Problemas derivados}

La problemática jurídica ligada a las CMSP no pasa únicamente por la interacción de los niveles de análisis y los destinatarios de estas normas. Existen al menos otros tres inconvenientes legales que serán trabajados a continuación: la tipificación de las CMSP y de su personal, la fuerza vinculante de las normas y la aplicabilidad de éstas.

En primer lugar, del marco jurídico hasta ahora explorado hay un referente internacional y uno doméstico. Del lado internacional está el Código de Conducta, el cual brinda algunas definiciones que pueden ser de utilidad para esta discusión como la de Compañías Privadas de Seguridad (PSCs, por sus siglas en inglés) y su personal. Las primeras pueden entenderse como

ualquier compañía (como es definida en el Código) cuyas actividades de negocios incluyan la provisión de servicios de seguridad (escolta y protección de personas y objetos, como convoyes, instalaciones, sitios designados, propiedades u otros lugares o cualquier otra actividad para la cual el personal de dichas compañías sea requerido para usar armas en el cumplimiento de su deber) a nombre propio o de otro, indistintamente de cómo esa compañía se describa a sí misma. ${ }^{19}$

El segundo es definido como

personas que trabajan para las PSCs, bien sea como empleados o bajo contrato, incluyendo sus operarios, gerentes y directores. Para evitar las dudas, las personas que son consideradas parte del personal de las PSCs es porque están conectadas con ellas a través de contratos de trabajo o por prestación de servicios, o si son contratistas independientes o trabajadores temporales o pasantes, independiente de la designación específica que le dé la compañía. ${ }^{20}$

A pesar de arriesgarse a dar una definición de compañías y su personal, el Código de Conducta demuestra dos grandes falencias. De un lado, restringe el universo de las compañías a aquellas que prestan servicios de escolta y protección, lo que pasa por alto a las empresas que ofrecen dentro de sus servicios operaciones militares y acciones ofensivas y, del otro, aunque define el personal de las compañías, nunca aclara su estatuto jurídico ${ }^{21}$.

Por el lado de lo doméstico aparece el acta sudafricana de 2006, la cuál es aún más modesta en materia de tipificación de lo que ya es el Código de Conducta. En ella, ni siquiera aparecen definidas las Compañías o el personal, dejando como único término de relevancia el de Servicios de Seguridad, que está ligado a las siguientes actividades: 
“a. protección o salvaguarda de un individuo, personal o propiedad de alguna manera, b. aconsejar acerca de la protección o salvaguarda de individuos o propiedades, c. aconsejar acerca del uso de equipo de seguridad, d. proveer un servicio de reacción o respuesta en conexión con la salvaguarda de personas o propiedad, e. proveer entrenamiento en seguridad o instrucción a un servicio de seguridad actual o prospectivo, f. instalación, acondicionamiento o reparación de equipo de seguridad, g. monitoreo o transmisiones desde equipos de seguridad". 22

La inexistencia de una tipificación pertinente y completa para las CMSP y su personal no obedece del todo a la incapacidad para entender este fenómeno por parte de las élites estatales o intergubernamentales. Prueba de ello son dos referentes (de nuevo uno universal y otro doméstico) sin fuerza legal, pero con mayor fondo conceptual.

Por una parte, se encuentra el borrador de la Convención Internacional sobre Regulación, Vigilancia y Monitoreo de las Compañias Militares y de Seguridad Privadas de 2009. Este borrador de convención internacional convocado por la ONU hace el esfuerzo por brindar definiciones más especializadas de lo que son las CMSP y del tipo de servicio que pueden prestar. Así, en su artículo 2 define Compañía Militar y/o de Seguridad Privada (CMSP) como "una entidad corporativa que provee sobre la base de la compensación servicios militares y/o de seguridad, incluidos servicios de investigación, por personas físicas y/o entidades legales"23. De otra parte, resulta interesante que este borrador hace la distinción entre Servicios Militares, los cuales "refieren a servicios especializados relacionados con acciones militares que incluyen planeación estratégica, inteligencia, investigación, reconocimiento aéreo o marítimo, operaciones de vuelo o de cualquier tipo, tripuladas o no tripuladas, vigilancia satelital, entrenamiento y logística militar, y apoyo técnico y material a las fuerzas armadas y otras actividades relacionadas" ${ }^{24}$ y Servicios de Seguridad que "refieren a vigilancia armada o protección de edificios, instalaciones, propiedad o personas, entrenamiento policial, apoyo técnico y material a las fuerzas de policía, elaboración e implementación de medidas de información en seguridad y otras actividades relacionadas" 25 .

El Green Paper hace un intento por definir las CMSP y distinguirlas de las Compañías Privadas de Seguridad. Las primeras se caracterizan por proveer fuerzas para el combate y ofrecen servicios de: asesoramiento, entrenamiento, apoyo logístico, oferta de personal para labores de monitoreo y desminado. El segundo tipo de compañías se distinguen por centrar sus servicios más en la provisión de servicios de seguridad en otros países para compañías o gobiernos. ${ }^{26}$

En segundo lugar, la fuerza vinculante con la que cuenta el marco jurídico disponible para regular el accionar de las CMSP y de su personal es muy débil. Esta debilidad se hace manifiesta en el sentido en que el grado de dureza de los instrumentos que tocan el tema de las CMSP polariza las posiciones de los potenciales destinatarios y termina inhibiendo su posible adhesión a los proyectos legales más vinculantes.

Como ya sido planteado, el marco jurídico existente es en preeminencia de soft law y que cuando se vislumbra un escenario viable de normas más de corte hard law se genera una tensión. Tensión que es palpable, por ejemplo, entre el documento de Montreux (soft law) y el borrador de convención (intento de hard law) por el enfoque que cada uno da al problema de las CMSP. Esta tensión entre marcos de regulación deriva en una confrontación normativa que trae como efecto el que los destinatarios opten por tomar partido por una de ellas dependiendo de sus intereses particulares, cosa que se cristaliza en la conformación de clientelas para cada acuerdo multilateral. Clientelas que marcan preferencias dadas las incompatibilidades entre provisiones sustantivas (en especial en lo que concierne a la discrepancia sobre lo que puede o no ser subcontratado a una CMSP). Así, los Estados con mayor conexión a la industria de las CMSP tendrá mejor recepción de lo que se adelante con el proceso de Montreux mientras los que se oponen a las CMSP como formas contemporáneas de mercenarismo de inclinan más por el proceso del borrador de la convención ${ }^{27}$.

Esta confrontación de visiones e intereses forja un estado de las cosas legitimador de un estatus quo en el que los Estados hegemónicos y las CMSP adoptan el espíritu del documento de Montreux y lo presentan 
como un esfuerzo importante para su rendición de cuentas, con la tranquilidad de no ser vinculante ni para los unos ni para las otras ${ }^{28}$.

En tercer lugar, y como fue señalado anteriormente, tanto el documento de Montreux como el Código de Conducta exhiben falencias en su aplicación. Estas falencias constan de la incapacidad de complementar al DIH existente y de contar con los medios para hacer acatar las buenas prácticas corporativas.

Al tener los mismos escenarios de aplicación del DIH, el documento de Montreux y el Código de Conducta están limitados por las circunstancias contempladas por este sistema normativo. Una limitación que va en dos direcciones: capacidad de regulación para los momentos de actuación de las CMSP y adjudicación de responsabilidad estatal.

El escenario de aplicación del DIH donde se patenta su incapacidad para regular el comportamiento de las CMSP es cuando no existe un conflicto armado declarado. La amplia gama de servicios ofertados por estas suele desplegarse en lugares en los que sus dirigentes no declaran un conflicto armado, sea interno o internacional. Así, la competencia del DIH como norma reguladora carece de validez ${ }^{29}$.

En el caso contrario, es decir, si el conflicto armado es declarado, el DIH brinda escasas posibilidades para establecer las obligaciones del Estado en el transcurso de la duración del contrato por prestación de servicios militares de seguridad. El DIH puede resultar aparentemente válido para pautar ciertas normas en el país donde las CMSP operen, lo cual tampoco sería sencillo si se toma en cuenta la complejidad de las acciones en las que el personal de las CMSP puede verse comprometido.

El documento de Montreux es incapaz de acompañar al DIH dado que presupone para su cumplimiento de derechos nacionales fuertes, que hagan respetar en su jurisdicción estándares internacionales de organización, supervisión y rendición de cuentas antes de otorgar una licencia. Montreux tampoco fija un patrón de conducta específica para que los Estados tengan una orientación en su proceder. Este documento se convierte así en una norma inoperante para sacar adelante legislaciones nacionales adecuadas ${ }^{30}$.

En el tema de mecanismos de cumplimiento, Montreux también se caracteriza por su inoperancia mientras el Código de Conducta se queda corto en su alcance. Por la parte de Montreux, este documento no hace alusión a la instalación de una oficina centralizada para registro de contratos, lo cual sería condición necesaria para garantizar la transparencia en la acción de las CMSP de cara a la opinión pública global y en particular a la que pertenece a los Estado donde ellas operan. Así, los documentos de Montreux podrían ser catalogados como una declaración de intenciones que fija estándares de buenas prácticas sin los instrumentos apropiados para hacerlas cumplir ${ }^{31}$. Por su parte, pese a que el Código de Conducta sí logra aterrizar los criterios de buen gobierno corporativo, las soluciones propuestas son incompletas para obligar a las compañías a la preservación de los DDHH. Por ende, ni Montreux ni el Código de Conducta componen una alternativa válida para conjurar las violaciones a los DDHH cometidas por miembros de las $\mathrm{CMSP}^{32}$.

\section{La necesidad de recurrir a otras figuras jurídicas}

Los notables vacíos jurídicos derivados de los distintos tipos de falencias expuestos por el marco jurídico, que aborda la cuestión de las CMSP en diferentes niveles, conllevan a pensar que es poco factible ver algún día a estas entidades corporativas o a su personal respondiendo penalmente ante tribunales internacionales como la Corte Penal Internacional. Con las CMSP en sí, es su condición de entidades legales lo que les permite gozar de una subjetividad discutible en el derecho internacional ${ }^{33}$. Con su personal, la situación de su estatuto hace incierta la normatividad aplicable para cada caso.

Ante el callejón sin salida aparente al que se ha llegado hasta este punto, se pretende formular una alternativa de solución que apunte a resolver el tema del estatuto de las CMSP, particularmente de su personal: explorar figuras jurídicas preexistentes en el derecho internacional que sean susceptibles de ser vinculadas con estos individuos. 


\section{El debate civil-combatiente-mercenario y las CMSP}

Tomando en cuenta los graves problemas en materia de tipificación y aplicación que tienen las CMSP y, por añadidura, su personal, pueden señalarse los siguientes elementos como ejes del debate para complementar a estos actores del derecho internacional: i) determinar la figura de "participación directa en las hostilidades" serviría para ubicar al personal de las CMSP como civiles o combatientes, ii) aunque el mercenarismo cuenta con un marco jurídico más sólido y con mayor fuerza vinculante, tipificar al personal de las CMSP como mercenarios dista bastante de carecer de problemas de aplicabilidad y vinculación, además de abrir una gran discusión entre similitudes y diferencias de ambas figuras y iii) el trasfondo de tal discusión excede el ámbito de lo jurídico y se desborda hacia el tema político.

\section{Civil-combatiente}

Un primer asunto por responder cuando se piensa en el estatus de los contratistas miembros de la CMSP es si ellos deben ser considerados como civiles o combatientes para los efectos del DIH. Ante la amplia gama de servicios prestados por las compañías a las que estos contratistas pertenecen, existe un acrecentamiento de la polémica legal en la medida en que este personal acompaña de forma más estrecha a los miembros de las fuerzas militares en el desarrollo de sus operaciones ${ }^{34}$.

$\mathrm{Al}$ regresar a los referentes del derecho internacional ya citados, para el documento de Montreux los miembros de las CMSP son civiles que pueden hacer parte de las hostilidades mientras que el borrador de la convención de la ONU discrepa sustancialmente de otorgarles dicha potestad ${ }^{35}$. Por lo tanto, se puede concluir parcialmente que el estatuto de los contratistas de las CMSP es una discusión que está lejos de estar resuelta, y que invita a establecer unas dimensiones de análisis como el contexto y la búsqueda de figuras jurídicas intermedias.

Por una parte, el debate acerca de la disyuntiva civil-combatiente se agudiza dependiendo del contexto en el que se desenvuelven. Si la participación de contratistas de CMSP se da en conflictos armados internacionales, existe la posibilidad de tipificarlos como combatientes con los derechos que este estatuto contrae. Si en cambio se está hablando de conflictos armados internos, esta posibilidad se diluye ante a la inexistencia de definición de combatiente para tal tipo de conflictos. Adicionalmente, el DIH relativo a los conflictos armados internos no contempla muchas herramientas para asociar a los contratistas de las CMSP con otras figuras jurídicas. De tal suerte que en la búsqueda de un estatuto singular para ellos deberá hacerse uso de dos tipos de fuentes: las convencionales (o que se desprenden de ellas) del derecho internacional concernientes a los conflictos internacionales, y los medios auxiliares como la doctrina científica.

Con respecto a las primeras, existen herramientas jurídicas útiles derivadas del derecho natural como la Cláusula Martens del II Convenio de la Haya de 1899 que va más allá del derecho convencional para evitar la inaplicabilidad del ius in bello a quienes participan en conflictos armados internacionales. Además, esta cláusula equipara a combatientes y civiles ante la ausencia de un derecho convencional al garantizar la protección y someterlos a los principios del derecho de gentes emanado de los usos establecidos ${ }^{36}$.

Siguiendo con el derecho convencional, el artículo 4 (A) (2) del III convenio de Ginebra extiende el estatuto de combatiente a milicianos o miembros de fuerzas voluntarias que cumplan las siguientes condiciones: "que estén bajo el mando de una persona responsable por sus subordinados, que porten signos distintivos reconocibles a la distancia, que porten armas abiertamente, que conduzcan las operaciones en acuerdo a las leyes y costumbres de la guerra" ${ }^{37}$. Esta definición extendida de combatiente inspirada en el convenio permitiría a los Estados que contratasen CMSP comprometidas en labores de combate para que se aseguren que los contratistas de éstas se acoplen a las disposiciones del artículo 4 (A) (2). Además, una 
expansión terminológica de esta envergadura también podría representar una deseable expansión hacia el ámbito de los conflictos armados internos ${ }^{38}$.

En la literatura académica se encuentran dos figuras intermedias que vale la pena cavilar: la de civiles que acompañan a las fuerzas armadas y la de no combatientes especiales. Defendiendo la primera postura se encuentran Shannon Bosch y Renée de Nevers quienes afirman que a pesar de que los contratistas de las CMSP no tengan la identificación que les brinde el estatuto de combatientes, esta figura los podría hacer acreedores de ciertas protecciones asociadas a dicho estatuto como, por ejemplo, lo ligado a la condición de prisioneros de guerra. ${ }^{39}$ Representando a la segunda postura, James Pattinson ofrece la categoría de no combatientes especiales, definidos como individuos que "participan en la empresa de la guerra, pero no participan directamente en acciones que conlleven a causar daño en los conflictos armados" ${ }^{40}$. Para este autor, esta categoría es más precisa que otras como "individuos parcialmente asimilados" en clases de combatientes porque ayuda a mantener una diferenciación entre los distintos roles que adoptan los combatientes y los no combatientes $^{41}$.

Hasta ahora, el derecho convencional y la doctrina científica han propuesto escenarios de equiparación entre combatientes y civiles (cláusula Martens), de ampliación del estatuto de combatiente (III convenio de Ginebra) y de puntos intermedios que parten de la figura de los contratistas como civiles con algunas características de combatientes sin acercarse del todo a este último estatuto. Sin embargo, las funciones que pueden terminar desempeñando los contratistas de las CMSP exigirían una tipificación más decidida por parte del derecho internacional.

El segundo asunto, que en últimas ayudaría a dar luces sobre la disyuntiva civil/combatiente para el personal de las CMSP, versaría en torno a la participación directa en las hostilidades. Ésta partiría de una relación causal directa y proporcional entre la actividad hostil desempeñada por los contratistas y el daño causado en el momento y lugar en el que la actividad tuviese lugar ${ }^{42}$. La participación en hostilidades se erigiría, entonces, como una alternativa más comprehensiva del accionar de los contratistas privados que resulta más sensible para los temas relativos al DIH y los DDHH. De ser válida la tesis de la participación directa en hostilidades, podría aplicarse mutatis mutandis; la definición de grupos armados que toman parte en un conflicto internacional al personal de las CMSP. ${ }^{43}$

\section{El problema del mercenarismo}

Resulta muy complejo determinar si los contratistas de las CMSP están más cerca al personal civil que al combatiente o viceversa. Las fuentes del derecho arriba utilizadas fuerzan la flexibilización de términos jurídicos, debilitando una hipotética aplicabilidad de obligaciones a estos contratistas. El escenario se hace aún más neurálgico ante la eventualidad de tipificar a estos individuos como combatientes. El debate que allí se abriría involucraría la recurrencia a una categoría mucha más desarrollada en el DIH: la del mercenarismo.

Sobre el mercenarismo acude un conjunto de referentes del derecho en los niveles universal, regional e interno. En el primer nivel aparece el derecho de Ginebra con el Protocolo Adicional I de 1977 y el derecho de Nueva York con las resoluciones de la Asamblea General de la ONU 2465 de 1968, 2548 de 1969 y 2708 de 1970 y del Consejo de Seguridad 239 de 1967, 405 de 1977 y 419 de 1977 que declaran punible el uso de mercenarios contra los movimientos de liberación nacional e independencia. Estas resoluciones serán el insumo de la Convención Internacional contra el reclutamiento, la utilización, la financiación yel entrenamiento de mercenarios de $1989^{44}$. Posteriormente, la resolución 58/162 del 22 de diciembre de 2003 y 2004/5 de la Comisión de Derechos Humanos del 8 de abril de 2004 harán lo propio con la resolución 2005/2 de la misma comisión, la cual: 
Insta a todos los Estados a que tomen las medidas necesarias y ejerzan la máxima vigilancia contra la amenaza que entrañan las actividades de los mercenarios y a que adopten medidas legislativas para asegurarse de que ni su territorio ni otros territorios bajo su control, ni sus nacionales, sean utilizados en el reclutamiento, la concentración, la financiación, el adiestramiento y el tránsito de mercenarios para planificar actividades encaminadas a obstaculizar el ejercicio del derecho de los pueblos a la libre determinación. ${ }^{45}$

En el nivel regional, el sistema africano se adelantó no solamente para regular, sino, incluso, para prohibir la actividad mercenaria en su territorio. En vez de quedarse en la mera denuncia ante los órganos de la ONU sobre la participación de mercenarios en sus guerras de descolonización (en especial décadas de 1960 y 1970), la Organización para la Unidad Africana (OUA) aprobó el Borrador para la Convención para la Eliminación de los Mercenarios en África de 1972. A pesar de que este borrador no logró la cantidad de Estados firmantes para entrar en vigor, el juicio de Luanda ${ }^{46}$ fue un evento decisivo para que el año siguiente la OUA tuviera una conquista en el frente antimercenario con la expedición de la Convención para la eliminación del mercenarismo en África.

En el nivel interno, la ley sudafricana No. 27 de 2006 para la prohibición de actividades mercenarias y regulación de ciertas actividades en paises con conflictos armados buscó la prohibición para exportar mercenarios, regular el aprovisionamiento de asistencia o servicio de naturaleza militar (o relacionado con éste) en un país con conflicto armado y regular el enrolamiento de ciudadanos sudafricanos en otras fuerzas $\operatorname{armadas}^{47}$.

Los instrumentos del derecho convencional acerca del mercenarismo recientemente citados aportan definiciones multidimensionales que ameritan un análisis sesudo. En primer lugar, el Protocolo Adicional I de Ginebra en su artículo 47 (2) entiende como mercenario toda persona:

a) que haya sido especialmente reclutada, localmente o en el extranjero, a fin de combatir en un conflicto armado;

b) que, de hecho, tome parte directa en las hostilidades;

c) que tome parte en las hostilidades animada esencialmente por el deseo de obtener un provecho personal y a la que se haga efectivamente la promesa, por una Parte en conflicto o en nombre de ella, de una retribución material considerablemente superior a la prometida o abonada a los combatientes de grado y funciones similares a las fuerzas armadas de esa Parte;

d) que no sea nacional de una Parte en conflicto ni residente en un territorio controlado por una Parte en conflicto;

e) que no sea miembro de las fuerzas armadas de una Parte en conflicto; y

f) que no haya sido enviada en misión oficial como miembro de sus fuerzas armadas por un Estado que no es Parte en conflicto. ${ }^{48}$

De su parte, la la Convención para la eliminación del mercenarismo en África hará lo propio en su artículo 1 al definir mercenario como una persona que:

a) Es reclutada local o exteriormente especialmente con el fin de combatir en un conflicto armado

b) Toma parte directamente en las hostilidades

c) Está motivada para tomar parte en las hostilidades esencialmente para obtener un lucro privado y de hecho una de las partes en conflicto, directamente o bajo su nombre, le promete una compensación material

d) No es nacional de una de las partes en conflicto ni tampoco es residente del territorio controlado por una de las partes en conflicto

e) No es miembro de las fuerzas armadas de una de las partes en conflicto

f) No es enviado por otro Estado diferente al que es una de las partes en conflicto en una misión oficial como parte de las fuerzas armadas de dicho Estado. ${ }^{49}$ 
Finalmente, la Convención Internacional contra el reclutamiento, la utilización, la financiación y el entrenamiento de mercenarios de 1989, también en su artículo 1, da la acepción de mercenario como toda persona:

a) Que haya sido especialmente reclutada, localmente o en el extranjero, para combatir en un conflicto armado;

b) Que tome parte en las hostilidades animada esencialmente por el deseo de obtener un provecho personal y a la que se haga efectivamente la promesa, por una Parte en conflicto o en nombre de ella, de una retribución material considerablemente superior a la prometida o abonada a los combatientes de grado y funciones similares en las fuerzas armadas de esa Parte;

c) Que no sea nacional de una Parte en conflicto ni residente en un territorio controlado por una Parte en conflicto;

d) Que no sea miembro de las fuerzas armadas de una Parte en conflicto; y

e) Que no haya sido enviada en misión oficial como miembro de sus fuerzas armadas por un Estado que no sea Parte en conflicto. ${ }^{50}$

Adicionalmente, esta última convención complementará su definición al entender también por "mercenario" toda persona en cualquier otra situación:

a) Que haya sido especialmente reclutada, localmente o en el extranjero, para participar en un acto concertado de violencia con el propósito de:

i. Derrocar a un gobierno o socavar de alguna otra manera el orden constitucional de un Estado, o de,

ii. Socavar la integridad territorial de un Estado;

b) Que tome parte en ese acto animada esencialmente por el deseo de obtener un provecho personal significativo y la incite a ello la promesa o el pago de una retribución material;

c) Que no sea nacional o residente del Estado contra el que se perpetre ese acto;

d) Que no haya sido enviada por un Estado en misión oficial; y

e) Que no sea miembro de las fuerzas armadas del Estado en cuyo territorio se perpetre el acto. ${ }^{51}$

Complementariamente, el relator especial para el mercenarismo de la Comisión de Derechos Humanos de las Naciones Unidas entre 1987 y 2004, Enrique Bernales Ballesteros, consideró a los mercenarios como:

\footnotetext{
las personas individuales que han sido recrutadas a través de organizaciones especiales para luchar por un Estado o grupos armados, y quien esta caracterizada por caracteristicas personales tales como habilidad y experiencia militar, ambición financiera, sentido de la aventura, deshumanización y tendencia a identificarse a si mismo con opciones ideologicas que tienen un componente autoritario. ${ }^{52}$
}

Si bien el mercenarismo tiene en a su disposición de una mayor cantidad de normas no solo regulatorias, sino también prohibitivas en comparación a las CMSP, tampoco ello se ha traducido en herramientas más efectivas de parte del derecho internacional. Los tratados aquí referenciados experimentan serias falencias para entablar responsabilidades y sanciones a los Estados y, sobre todo, a las CMSP.

El tratado más problemático para la tipificación del mercenarismo es el Protocolo Adicional I de Ginebra. Las objeciones al protocolo respecto al asunto de los mercenarios guarda relación con a) la exigencia de contar con cada uno de los seis elementos de definición si se quiere prescribir una conducta delictiva, b) su improcedencia por imposibilidad de probar la motivación de alguien acusado de actividades mercenarias, c) dejar a un lado al personal militar extranjero que integra las fuerzas armadas de otro Estado y a los extranjeros empleados como consejeros militares de un Estado y d) el excluir al mercenarismo de la discusión hacia conflictos armados internos. ${ }^{53}$ 
Aunque la Convención para la eliminación del mercenarismo en África pareciera superar las limitaciones del protocolo al encuadrar al mercenarismo como un delito en sí, independiente de otras ofensas, persisten en esta primera otras cuestiones difíciles de resolver. La convención aspira a generalizar el crimen de mercenarismo a individuos, grupos u organizaciones que representan al Estado o son del Estado y que acogen, organizan, financian, enlisten bandas de mercenarios. La convención africana tendría entonces la potestad para ligar a los contratistas de las CMSP con actividades mercenarias. Sin embargo, y como en el protocolo, las disposiciones de la convención se aplican a conflictos armados internacionales, una desventaja si se entiende que la mayor parte de las actividades mercenarias se desarrollan en conflictos no internacionales ${ }^{54}$.

Por último, en la Convención Internacional contra el reclutamiento, la utilización, la financiación y el entrenamiento de mercenarios el asunto se complica por el lado del proceso de ratificación del tratado y otra vez por la definición de mercenario. Circunstancia que responde en buena medida a dos inconvenientes. El primero de ellos, al escaso apoyo de los Estados parte para impulsar el instrumento. Solamente 26 Estados han ratificado este tratado. La falta de prisa de los líderes estatales para erradicar el mercenarismo y la utilización de CMSP y un número más creciente de Estados son las dos razones más plausibles para explicar esta apatía ${ }^{55}$. El segundo inconveniente radica en la imposibilidad de imputar a un supuesto mercenario basado en la definición de esta convención, puesto que allí se define al mercenario como un individuo en lugar de como una actividad. Por ende, al plantearse el beneficio económico como el principal motivo del mercenario, se hace complicado probar una relación causal directa entre ambas cosas ${ }^{56}$.

En resumen, aunque la figura jurídica del mercenarismo tenga una tipificación más desarrollada en el derecho internacional en contraste con las CMSP y su personal, ambos términos comparten sus falencias fundamentales en términos de aplicabilidad y fuerza vinculante, mayormente en lo que tiene que ver con conflictos armados internos. Falta observar hasta qué punto ambos conceptos son mutuamente asimilables.

\section{Mercenarismo y CMSP}

La viabilidad de ligar a los contratistas de las CMSP al estatuto de mercenarios va más allá de lo retórico y es un debate cuyas raíces se hunden profundamente en los ámbitos de lo jurídico y lo histórico. Resulta pertinente entonces explorar las diferencias y similitudes de fondo entre ambas categorías.

En lo que concierne a las diferencias, János Kálmán hace un esfuerzo por entablar las principales distinciones que tienen las CMSP de los mercenarios, las cuales se desprenden de la naturaleza y el accionar propios de las unas y los otros (ver Tabla 1).

TABLA 1

\begin{tabular}{|l|l|l|}
\hline Mercenarios & Características & CMSP \\
\hline $\begin{array}{l}\text { Tratar de estar alejados de } \\
\text { la atención pública }\end{array}$ & Publicidad & $\begin{array}{l}\text { Mensajes comerciales } \\
\text { transparentes ofreciendo } \\
\text { sus servicios }\end{array}$ \\
\hline $\begin{array}{l}\text { Pequeños grupos de } \\
\text { individuos }\end{array}$ & Forma de org anización & $\begin{array}{l}\text { Corporativa, estructura de } \\
\text { negocio clara, personeria } \\
\text { jurídica }\end{array}$ \\
\hline $\begin{array}{l}\text { Usualmente ciudadanos } \\
\text { extranjeros, terroristas, } \\
\text { criminales, guerrillas }\end{array}$ & Miembros & $\begin{array}{l}\text { Los miembros usualmente } \\
\text { han servido previamente } \\
\text { en ejércitos nacionales, y } \\
\text { son reclutados desde bases } \\
\text { de datos multinacionales }\end{array}$ \\
\hline $\begin{array}{l}\text { De "abajo hacia arriba" } \\
\text { (Bottom-up) }\end{array}$ & $\begin{array}{l}\text { Forma de privatización de } \\
\text { la violencia }\end{array}$ & $\begin{array}{l}\text { De arriba hacia abajo "Top- } \\
\text { down" }\end{array}$ \\
\hline $\begin{array}{l}\text { Actividad directa ofensiva } \\
\text { Actividad }\end{array}$ & $\begin{array}{l}\text { Entrenamiento, asesoría } \\
\text { profesional, } \\
\text { contraespionaje, } \\
\text { mantenimiento, apoyo } \\
\text { logistico y militar, apoyo en } \\
\text { vigilancia militar o } \\
\text { reconstrucción posconflicto }\end{array}$ \\
\hline $\begin{array}{l}\text { Mercado negro, pago en } \\
\text { efectivo }\end{array}$ & $\begin{array}{l}\text { Condiciones de } \\
\text { contratación }\end{array}$ & $\begin{array}{l}\text { Contratos, muchas veces } \\
\text { licitación pública }\end{array}$ \\
\hline
\end{tabular}

Fuente: János Kálmán., op. cit., 375.

Quienes abogan por la diferenciación entre mercenarios y CMSP parten por distinguir los modos de organización y contratación en cada caso. En los primeros, la estructura es menos complicada, la contratación 
es informal e incluso ilegal. Las segundas se precian de tener un gobierno corporativo, una jerarquía definida y un registro conforme a la ley estatal en donde operen. ${ }^{57}$

Desde la perspectiva del accionar de caso uno, el talante entre acciones ofensivas y defensivas marca la diferencia para ambas figuras. En el caso de los mercenarios, la acción armada es directa y es ofensiva según la Tabla 1. Para las CMSP se impone la acción defensiva y de protección de personal, infraestructura e inclusive de personal humanitario como los miembros de la ONU, otras organizaciones internacionales, CICR y ONGs. Además, el nivel de especialización de sus contratistas les permite incluso fungir como guardianes de la paz en caso de que las instancias multilaterales voluntarias sean insuficientes o incapaces ${ }^{58}$.

En lo que respecta a la literatura académica que se enfoca en encontrar más similitudes que diferencias entre mercenarios y CMSP es necesario señalar que ellos piensan que la necesidad de disociar ambos conceptos refleja en buena parte un discurso legitimador de las segundas. Una primera cuestión que debe ser desvelada de ese discurso es el del mercenarismo como manifestación del afán de lucro individual y de los contratistas de las CMSP como miembros de un cuerpo colectivo con un mando centralizado. Lo categórico de este argumento les resulta falaz tanto jurídica como históricamente. Del lado jurídico, aunque el Protocolo Adicional I de Ginebra y en cierto modo la Convención Internacional contra el reclutamiento, la utilización, la financiación y el entrenamiento de mercenarios centran su definición de mercenario en individuos aislados, fortificando la postura de quienes consideran que los dos conceptos en discusión son incompatibles, la Convención para la eliminación del mercenarismo en África enlaza su definición a grupos y empresas. Del lado histórico, instituciones derivadas del mercenarismo como los condottieri medievales o las Compañías neerlandesa o británica de las Indias orientales plasman el talante tanto colectivo como corporativo que puede asumir el mercenarismo. Por lo tanto, la naturaleza de unos y otros no es tan disímil como se querría hacer pensar.

En lo competente a la relación entre fuerzas defensivas y fuerzas ofensivas, los defensores de la asociación vuelven a basarse en la historia. Por ejemplo, los disociadores desconocen el papel y el accionar de los mercenarios suizos en la Francia de Luis XVI, al aseverar que las CMSP rompen con los mercenarios en tanto son fuerzas de "seguridad defensiva o pasiva" 59 .

Una segunda cuestión que asocia a los contratistas de las CMSP con el accionar mercenario es la postura de sociedades periodísticas y representantes de organizaciones internacionales ante la disociación mencionada. En la literatura académica sobre la materia son varios los autores que sin mayores prevenciones consideran al personal de las CMSP como mercenarios contemporáneos. El International Consortium of Investigative Journalists considera la categoría CMSP como un eufemismo para referirse a los mercenarios ${ }^{60}$. Mención especial merece el anteriormente citado relator especial sobre mercenarismo, Bernales Ballesteros, quien sin dilaciones calificó a empresas como Executive Outcomes en Angola como mercenarios. El hecho de que estas empresas cobren por sus actividades y tengan conexión con gobiernos corruptos y empresas multinacionales que desean hacer negocios lucrativos con los recursos naturales de los países pobres han sido las razones por las que Bernales osó llamar a las CMSP "nuevas modalidades de mercenarismo" ${ }^{61}$.

La intención de disociación entre CMSP y mercenarios parte de unos intereses creados por líderes políticos y encarna un debate que debe partir de este supuesto. Una eventual tipificación vinculante y universal que cobije a las CMSP como mercenarios podría poner en riesgo la presencia de estas compañías en más de 50 países donde estas operan el día de hoy. Los efectos de una acción así afectarían un negocio billonario, así como la manutención o búsqueda de la seguridad de potencias globales y regionales y de sus Estados clientes.

Antes que nada, este es un problema político que responde a un orden mundial específico y a un modelo económico específico: el neoliberal. El modelo neoliberal se manifiesta como una disposición del sistema internacional para moldear las elecciones de los tomadores de decisión de las grandes potencias y derivadamente de sus contrapartes en la periferia ${ }^{62}$.

El constreñimiento a las elites no se da solamente en el ámbito intraestatal, sino que permea el seno mismo de las organizaciones internacionales como la ONU. Como ha sido referido, el peruano Enrique 
Bernales Ballesteros tuvo una actitud valiente al ligar a las CMSP con el mercenarismo. Bernales había tomado una postura clara al acusar a las CMSP como mercenarios, una postura que la mayoría de la comunidad internacional había elegido rechazar. Ante el cambio progresivo de contexto desde que Bernales asumió la relatoría en 1987 y la profundización del neoliberalismo, un primer constreñimiento constaría en hacerlo moderar su posición. En una visita al Reino Unido, Bernales había reconocido públicamente la necesidad que tenías estas compañías para trabajar. No obstante, su resistencia a desvincular a las CMSP a las convenciones sobre mercenarismo requería tomar medidas más contundentes ${ }^{63}$.

El reemplazo en 2004 de Bernales por Shaista Shameem significó un cambio sustantivo de opinión desde la oficina del relator especial, permitiendo una implementación muy laxa de las dos convenciones antimercenarios universales. De esa manera, la disociación entre CMSP y mercenarismo logró cristalizarse por la vía del derecho consuetudinario internacional a través de las prácticas Estados-compañías ${ }^{64}$.

En síntesis, un análisis integral del fenómeno de las CMSP y de su repercusión sobre los principios del derecho internacional debe trascender el campo de lo estrictamente jurídico y entender una realidad política e internacional más compleja que tiende al inmovilismo. Inmovilismo que, en última instancia, favorece un status quo provechoso para los poderosos y perjudicial para los débiles.

\section{Conclusión}

Tomando en cuenta que la idea central de este artículo consideraba que la debilidad que exhibe el derecho internacional para dar herramientas de regulación a los contratistas de las CMSP o para vincularlos a otras figuras jurídicas mejor tipificadas refleja un problema eminentemente político que alimenta un status quo antidemocrático, puede decirse que hay una demostración parcial de este argumento.

Las falencias del derecho internacional son fuertes si se toma en consideración que los acuerdos existentes sobre CMSP privilegian al soft law, a las reglas del DIH y de los DDHH ya ratificadas y a las normas del derecho interno más de los países en donde estas compañías tienen sus centros de operaciones que de aquellos en donde ellas operan. Además, si se piensa en que el derecho internacional vigente debe cumplir una serie de condiciones para tener competencias sobre el actuar de estos individuos, su papel se limita aún más. Estas condiciones pasarían por la declaración de conflicto, la discriminación entre conflictos armados internos, conflictos internacionales y por el estatuto jurídico del personal de las CMSP.

Con respecto a dicho estatuto, los problemas se acrecientan en la medida en que cualquier intento de regulación de las CMSP deba superar las graves dificultades observables con respecto a la tipificación, aplicabilidad y fuerza vinculante que caracteriza al marco jurídico sobre estas compañías y sus contratistas.

No obstante, recurrir a otra figura jurídica tampoco resolvería el impasse que plantean estos actores del derecho internacional. Las figuras propuestas en este artículo se desprenden de la flexibilización de normas del DIH y de proposiciones de la doctrina científica. Esta situación deja como camino viable la escogencia del término mercenario para complementar la visión jurídica sobre los contratistas militares privados. Esta escogencia logra superar mínimamente las exigencias de tipificación, pero agudiza los problemas de aplicabilidad y fuerza vinculante. Sin embargo, las razones para que esto sea así exceden los ámbitos de lo jurídico y lo histórico para apostarse preeminentemente en el ámbito de lo político.

Las alternativas de solución para esta problemática urgen no solamente para perfeccionar al derecho internacional. Los vacíos jurídicos y la ausencia de herramientas de regulación de las compañías estudiadas vulneran los principios mismos del derecho internacional, lo que contraviene la moral universal e ignora el dolor de las poblaciones más frágiles. 


\section{Bibliografía}

Benjamin Perrin, Mind the Gap: Lacunae in the International Legal Framework Governing Private Military and Security Companies, 31 Criminal Justice Ethics, n. ${ }^{\circ} 3$, 213-232, (2012). https://doi.org/10.1080/0731129X.2012.7371 70

Christopher Kinsey, Challenging international law: a dilemma of private security companies, 5 Conflict, Security \& Development, n. ${ }^{\circ} 3,269-293$ (2005). https://doi.org/10.1080/14678800500344515

Christopher Kinsey, Coporate soldiers and International Security (Routledge, 2006). https://doi.org/10.4324/9780 203018354

Clive Walker, \& Dave Whyte, Contracting out War?: Private Military Companies, Law and Regulation in the United Kingdom, 54 The International and Comparative Law Quaterly, n. ${ }^{\circ}$ 3, 651-689 (2005). https://doi.org/10.109 3/iclq/lei021

Daniel Warner, Establishing norms for Private Military and Security Companies, 40 Denver Journal of International Law \& Policy, n. ${ }^{\circ} 1,106-117$ (2011). https://digitalcommons.du.edu/cgi/viewcontent.cgi?article=1143\&cont ext $=$ djilp

Elise Rousseau, International Law and Private Military Companies: a Search for Clarity Droit International, sociétés militaires et conflit armé: Entre incertitudes et responsabilités, 8 Journal of Intervention and Statebuilding, n. ${ }^{\circ}$, 99-104 (2014). https://doi.org/10.1080/17502977.2014.877624

Elke Krahmann, From 'Mercenaries' to 'Private Security Contractors': The (Re) Construction of Armed Security Providers in International Legal Discourse, 40 Millenium: Journal of international Studies, n. ${ }^{\circ} 2,343-363$ (2012). https:/ /doi.org/10.1177/0305829811426673

Faiza Patel, Regulating Private Military and Security Companies: A comprehensive solution, 107 ASIL Proceedings, 201-203 (2013). https://doi.org/10.5305/procannmeetasil.107.0201

Fred Schreier \& Marina Caparini, Privatising Security: Law, Practice and Governance Private Military and Security Companies (Geneva Centre for the Democratic Control of Armed Forces, 2005).

James Pattison, The legitimacy of the military, private military and security companie, and just war, 11 European Journal of Political Theory, n. ${ }^{\circ} 2,131-154$ (2011). https://doi.org/10.1177/1474885111425119

János Kálmán, Mercenaries Reloaded? Applicability of the Notion of 'Mercenaries' in Relation to Private Military Companies and their Employees, 54 Acta Juridica Hungarica, n. ${ }^{\circ} 4,367-383$ (2013). https://doi.org/10.1556/a jur.54.2013.4.5

Jenny S. Lam, Accountability for Private Military Contractors under the Alien Tort Statute, 97 California Law Review, n. ${ }^{\circ}$ 5, 1459-1499 (2009). https://www.business-humanrights.org/en/latest-news/pdf-accountability-for-privat e-military-contractors-under-the-alien-tort-statute/

José L. Gómez del Prado, Private Military and Security Companies and the UN Working Group on the Use of Mercenaries, 13 Journal of Concflict \& Security Law, n. ${ }^{\circ}$, 429-450 (2009). https://doi.org/10.1093/jcsl/krp 010

Joseph C. Hansen, Rethinking the regulation of Private Military and Security Companies under International Humanitarian Law, 35 Fordham International Law Journal, n. ${ }^{\circ}$ 3, 698-736 (2012). https://ir.lawnet.fordham. $\mathrm{edu} / \mathrm{cgi} / \mathrm{viewcontent}$.cgi?article $=2437 \&$ context $=\mathrm{ilj}$

Julio Jorge Urbina, Actores no estatales y externalización de las funciones militares en los conflictos armados: los contratistas privados ante el derecho internacional humanitario, 8 ACDI, $41-85$ (2015). https://doi.org/10.12804/acdi8.1. 2015.02

Kristine A. Huskey, Accountability for Private Military and Security Contractors in the International Legal Regime,31 Criminal Justice Ethics, n. 3 3, 193-212 (2012). https://doi.org/10.1080/0731129X.2012.737169

Marcus Hedahl, Unaccountable: The Current State of Private Military and Security Companies, 31 Criminal Justice Ethics, n. ${ }^{\circ} 3,175-192$ (2012). https://doi.org/10.1080/0731129X.2012.740907 
Meg Roggensack, U.S. Legislative and Regulative Developments and the International Code of Conduct for Private Security Providers: Filling the Accountability Gap?, 107 ASIL Proceedings, 203-204 (2013). https://doi.org/10. 5305/procannmeetasil.107.0203

Michael N. Schmitt, Humanitarian Law and Direct Participation in Hostilities by Private Contractors or Civilian Employees, 5 Chicago Journal of International Law, n. 2 , $511-546$ (2005). https://chicagounbound.uchicago.e $\mathrm{du} / \mathrm{cjil} / \mathrm{vol} 5 / \mathrm{iss} 2 / 11$

Mohamad Ghazi Janaby, The Legal Status of Employees of Private Military/Security Companies Participating in U.N. Peacekeeping Operations, 13 Northwestern Journal of International Human Rights, n. ${ }^{\circ}$ 1, 82-102 (2015). https: //scholarlycommons.law.northwestern.edu/njihr/vol13/iss1/4/

Nigel D. White, The Privatisation of Military and Security Functions and Human Rights: Comments on the UN Working Group's Draft Convention, 11 Human Rights Law Review, n. ${ }^{\circ}$ 1, 133-151 (2011). https://doi.org/10.1093/hrlr /ngq053" https://doi.org/10.1093/hrlr/ngq053

Paul Jackson, 'War is much too serious a thing to be left to military men': Private military companies, combat and regulation, 5 Civil Wars, n. ${ }^{\circ}$ 4, 30-55 (2002). https://doi.org/10.1080/13698240208402516

Pilar Pozo Serrano \& Maria Lourdes Hernández Martín, El Marco Jurídico de las CMSP. Reflexiones a propósito de la experiencia en Irak, 23 Anuario Español de Derecho Internacional, 315-351 (2007). http://eprints.lse.ac.uk/1 $01217 /$

Renée De Nevers, Private Security Companies and the Laws of War, 40 Security Dialogue, n. ${ }^{\circ}$ 2, 169-190 (2009). ht tps://doi.org/10.1177/0967010609103076

Ricardo Abello Galvis, Introducción al estudio de las normas de ius cogens en el seno de la comisión de derecho internacional, 60 Vniversitas, n. ${ }^{\circ}$ 123, 75-104 (2011). https://doi.org/10.11144/Javeriana.vj60-123.ieni" https://doi.org/10.11144/Javeriana.vj60-123.ieni

Shannon Bosch, Private security contractors and international bumanitarian law - a skirmish for recognition in international armed conflicts, 16 African Security, n. ${ }^{\circ} 4,34-52$ (2007). https://doi.org/10.1080/10246029.200 7.9627443

Thomas K. Adams, Private Military Companies: Mercenaries of the 21 st Century, 13 Small Wars and Insurgencies, n. 2, 54-67 (2002). https://doi.org/10.1080/09592310208559181

Todd S. Milliard, Overcoming Post-Colonial Myopia: a call to recognize and regulate Private Military Companies, 176 Military Law Review, 1-96 (2003). https://apps.dtic.mil/dtic/tr/fulltext/u2/a440071.pdf

Private security companies Under-regulated growth, 11 Strategic Comments, 1-2 (2007). https://doi.org/10.1080/13 56788051105

Geoffrey S. Corn, Contractors and the Law, en Contractors and War: The Transformation of United States' Expeditionary Operations, 157-183 (Christopher Kinsey \& Malcom Hugh Patterson eds., Stanford University Press, 2012).

Jennifer K. Elsea \& Nina M. Serafino, Private Security Contractors in Iraq: Background, Legal Status and Other Issues, en Defense, Security and Strategies: Security in Iraq, 139-169 (James L. Jones, Jennifer K. Elsea \& Nina M. Serafino, Nova, 2010).

Ulrich Petersohn, Reframing the anti-mercenary norm: Private military and security companies and mercenarism, 69 International Journal, n. ${ }^{\circ}$ 4, 475-493 (2014). https://www.jstor.org/stable/24709418

au.int/AHG/49(IV), Resolución 49(IV) de la Organización para la Unidad Africana "sobre las actividades de los mercenarios".

United Kingdom, Foreign and Commonwealth Office, HC 577 Private Military Companies: Options for Regulation 2001-02 (2002). https://assets.publishing.service.gov.uk/government/uploads/system/uploads/attachment_d ata/file/228598/0577.pdf

Confederación Suiza, The International Code of Conduct for Private Security Service Providers (2010). https://icoca.ch/ sites/all/themes/icoca/assets/icocenglish3.pdf" https://icoca.ch/sites/all/themes/icoca/assets/icocenglish3.pdf

CICR \& Confederación Suiza, Dirección de Derecho Internacional del Departamento Federal de Relaciones Exteriores, Documento de Montreux sobre las obligacionesjurídicas internacionalespertinentes y las buenas prácticas 
de los Estados en lo que respecta a las operaciones de las empresas militares y de seguridad privadas durante los conflictos armados (2008). https://www.icrc.org/es/publication/documento-de-montreux-sobre-las-empres as-militares-y-de-seguridad-privadas" https://www.icrc.org/es/publication/documento-de-montreux-sobre-las -empresas-militares-y-de-seguridad-privadas

Protocolo I adicional a los Convenios de Ginebra de 1949 relativo a la protección de las víctimas de los conflictos armados internacionales (1977). https://www.icrc.org/es/document/protocolo-i-adicional-convenios-ginebra1949-proteccion-victimas-conflictos-armados-internacionales-1977

Convención Internacional contra el reclutamiento, la utilización, la financiación y el entrenamiento de mercenarios (1989). https://www.icrc.org/spa/resources/documents/misc/treaty-1989-mercenaries-5tdmhy.htm

CICR \& Confederación Suiza, Montreux +5 Conference (2013). https://www.icrc.org/eng/resources/documents/ legal-fact-sheet/private-military-companies-montreux-plus-5-conclusions.htm" https://www.icrc.org/eng/reso urces/documents/legal-fact-sheet/private-military-companies-montreux-plus-5-conclusions.htm

Act No. 27 of 2006, República de Sudáfrica, Probibition of Mercenary Activities and Regulation of Certain Activities in Country of Armed Conflict Act", Government Gazette (2007). http://www.ohchr.org/Documents/Issues/Merc enaries/WG/Law/SouthAfrica2.pdf

Naciones Unidas, Oficina del Alto Comisionado de las Naciones Unidas para los Derechos Humanos, Draft International Convention on the Regulation, Oversight and Monitoring of Private Military and Security Companies (2009). http://www2.ohchr.org/english/issues/mercenaries/docs/A.HRC.15.25.pdf

undocs.org/ A/60/263. Naciones Unidas, Asamblea General, Uso de mercenarios como un medio para la violación de derechos humanos e impedir el ejercicio al derecho de los pueblos a la libre determinación (2005).

\section{Notas}

* Artículo de investigación.

1 Documento de Montreux sobre las obligaciones jurídicas internacionales pertinentes y las buenas prácticas de los Estados en lo que respecta a las operaciones de las empresas militares y de seguridad privadas durante los conflictos armados, firmado el 17 de septiembre de 2008 y que contó con el concurso de 17 Estados: Afganistán, Alemania, Angola, Australia, Austria, Canadá, China, Estados Unidos de América, Francia, Irak, Polonia, Reino Unido de Gran Bretaña e Irlanda del Norte, Sierra Leona, Sudáfrica, Suecia, Suiza y Ucrania. Este documento "es el fruto de un proceso internacional iniciado por el Gobierno suizo y el CICR. Es un documento intergubernamental destinado a la promoción del respeto del derecho internacional humanitario y del derecho internacional de los derechos humanos en todos los conflictos armados donde intervengan empresas militares y de seguridad privadas". CICR \& Confederación Suiza, Dirección de Derecho Internacional del Departamento Federal de Relaciones Exteriores, Documento de Montreux sobre las obligaciones juridicas internacionales pertinentes y las buenas prácticas de los Estados en lo que respecta a las operaciones de las empresas militares $y$ de seguridad privadas durante los conflictos armados (2008).

2 Ricardo Abello Galvis, Introducción al estudio de las normas de ius cogens en el seno de la comisión de derecho internacional, 60 Vniversitas, . $^{\circ} 123,75-104$ (2011).

3 Íd.

4 CICR \& Confederación Suiza, Dirección de Derecho Internacional del Departamento Federal de Relaciones Exteriores, Documento de Montreux sobre las obligaciones jurídicas internacionales pertinentes y las buenas prácticas de los Estados en lo que respecta a las operaciones de las empresas militares y de seguridad privadas durante los conflictos armados (2008).

5 CICR \& Confederación Suiza, Montreux +5 Conference (2013).

6 Confederación Suiza, The International Code of Conduct for Private Security Service Providers (2010).

7 Private security companies Under-regulated growth, 11 Strategic Comments, 1-2 (2007).

8 Íd.

9 Fred Schreier \& Marina Caparini, Privatising Security: Law, Practice and Governance Private Military and Security Companies, 107-108 (Geneva Centre for the Democratic Control of Armed Forces, 2005).

10 Según Meg Roggensack, el derecho estadounidense ofrece tres desarrollos a este respecto: el Civilian Extraterritorial Jurisdiction Act (expansión y clarificación de la jurisdicción criminal de los contratistas federales y empleados en el exterior para cualquier propósito), la Executive Order 13627 (fortalecer la protección para trata de personas en contratos federales) y el End Trafficking in Government Contracting Act (contratar personal de países de bajos salarios) Meg Roggensack, U.S. Legislative and Regulative Developments and the International Code of Conduct for Private Security 
Providers: Filling the Accountability Gap?, 107 ASIL Proceedings, 203-204 (2013). Por su parte, Jennifer K. Elsea \& Nina M. Serafino, parten de establecer un marco jurídico para las CMSP más desde la legislación militar, partiendo de la base de que el personal de las primeras realiza labores de apoyo a los individuos regulados por la segunda. Para ellas, la sección 522 del acto de autorización de defensa nacional Jhon Warner FY2007(PL 109-364) hacen de los contratistas privados que trabajan en Irak y apoyan a las fuerzas militares objeto de las cortes marciales, pero hasta que el Departamento de Defensa publique regulaciones, es posible que los contratistas que cometan crimenes en Irak sean juzgados bajo el estatuto criminal que aplica extra territorialmente o bajo la jurisdicción especial marítima o terrestre de USA o por el acto de la jurisdicción militar extraterritorial (MEJA). Jennifer K. Elsea \& Nina M. Serafino, Private Security Contractors in Iraq: Background, Legal Status and Other Issues, en Defense, Security and Strategies: Security in Iraq, 139-169 (James L. Jones, Jennifer K. Elsea \& Nina M. Serafino, Nova, 2010).

11 Meg Roggensack, op. cit., 107, 203-204.

12 Jenny S. Lam, Accountability for Private Military Contractors under the Alien Tort Statute, 97 California Law Review, n. ${ }^{\circ} 5,1459-1499$ (2009).

13 Strategic comments, op. cit.; Marcus Hedahl, Unaccountable: The Current State of Private Military and Security Companies, 31 Criminal Justice Ethics, n. 3, 175-192 (2012); Jennifer K. Elsea \& Nina M. Serafino, op. cit.

14 Jenny S. Lam, op. cit.

15 Christopher Kinsey, Challenging international law: a dilemma of private security companies, 5 Conflict, Security \& Development, n. ${ }^{\circ 3}, 269-293$ (2005).

16 CICR \& Confederación Suiza, op. cit.

17 CICR \& Confederación Suiza, op. cit.

18 Confederación Suiza, op. cit.

19 Íd.

20 Íd.

21 Daniel Warner, Establishing norms for Private Military and Security Companies, 40 Denver Journal of International Law \& Policy, n. ${ }^{\circ} 1,106$ (2011).

22 Act No. 27 of 2006, República de Sudáfrica, Prohibition of Mercenary Activities and Regulation of Certain Activities in Country of Armed Conflict Act", Government Gazette (2007).

23 Naciones Unidas, Oficina del Alto Comisionado de las Naciones Unidas para los Derechos Humanos, Draft International Convention on the Regulation, Oversight and Monitoring of Private Military and Security Companies (2009).

24 Íd.

25 Íd.

26 United Kingdom, Foreign and Commonwealth Office, HC 577 Private Military Companies: Options for Regulation 2001-02 (2002).

27 Nigel D. White, The Privatisation of Military and Security Functions and Human Rights: Comments on the UN Working Group's Draft Convention, 11 Human Rights Law Review, n. ${ }^{\circ}$ 1, 133-151 (2011).

28 Elise Rousseau, International Law and Private Military Companies: a Search for Clarity Droit International, sociétés militaires et conflit armé: Entre incertitudes et responsabilités, 8 Journal of Intervention and Statebuilding, n. ${ }^{\circ}$ 1, 99-104 (2014).

29 Kristine A. Huskey, Accountability for Private Military and Security Contractors in the International Legal Regime, 31 Criminal Justice Ethics, n. ${ }^{\circ}$ 3, 201 (2012).

30 Nigel D. White, op. cit., 136-137.

31 José L. Gómez del Prado, Private Military and Security Companies and the UN Working Group on the Use of Mercenaries, 13 Journal of Concflict \& Security Law, n. ${ }^{\circ}$ 3, 441-447 (2009).

32 Faiza Patel, Regulating Private Military and Security Companies: A comprehensive solution, 107 ASIL Proceedings, 201-203 (2013).

33 Benjamin Perrin, Mind the Gap: Lacunae in the International Legal Framework Governing Private Military and Security Companies, 31 Criminal Justice Ethics, n.. 3, 228 (2012).

34 Geoffrey S. Corn, Contractors and the Law, en Contractors and War: The Transformation of United States' Expeditionary Operations, 157-183 (Christopher Kinsey \& Malcom Hugh Patterson eds., Stanford University Press, 2012).

35 Nigel D. White, op. cit., 138.

36 Julio Jorge Urbina, Actores no estatales y externalización de las funciones militares en los conflictos armados: los contratistas privados ante el derecho internacional humanitario, 8 ACDI, 48 (2015).

37 Shannon Bosch, Private security contractors and international humanitarian law - a skirmish for recognition in international armed conflicts, 16 African Security, n. ${ }^{\circ}$ 4, 37 (2007). 
38 Joseph C. Hansen, Rethinking the regulation of Private Military and Security Companies under International Humanitarian Law, 35 Fordham International Law Journal, n. 3, 736 (2012).

39 Shannon Bosch, op. cit., 40-41; Renée De Nevers, Private Security Companies and the Laws of War, 40 Security Dialogue, n. ${ }^{\circ}$ 2, 175 (2009).

40 James Pattison, The legitimacy of the military, private military and security companie, and just war, 11 European Journal of Political Theory, n. ${ }^{\circ}$ 2, 131-154 (2011).

41 Íd., 151.

42 Michael N. Schmitt, Humanitarian Law and Direct Participation in Hostilities by Private Contractors or Civilian Employees, 5 Chicago Journal of International Law, n. 2, $511-546$ (2005); János Kálmán, Mercenaries Reloaded? Applicability of the Notion of'Mercenaries' in Relation to Private Military Companies and their Employees, 54 Acta Juridica Hungarica, n. ${ }^{\circ}$ 4, 379 (2013).

43 Julio Jorge Urbina, op. cit., 54.

44 Convención Internacional contra el reclutamiento, la utilización, la financiación y el entrenamiento de mercenarios (1989).

45 undocs.org/ A/60/263. Naciones Unidas, Asamblea General, Uso de mercenarios como un medio para la violación de derechos humanos e impedir el ejercicio al derecho de los pueblos a la libre determinación (2005).

46 En el año de 1976 en la capital de Angola fueron juzgados y sentenciados por crímenes de guerra 13 mercenarios británicos y norteamericanos, nueve de los cuales recibieron prolongadas condenas de prisión y los otros cuatro fueron condenados a muerte. Como efecto del juicio, el Estado angoleño convocó una comisión internacional cuyos delegados provenían principalmente de países tercermundistas y del bloque oriental. Tal comisión condenó el mercenarismo como "parte de un proceso de perpetuación por la fuerza de las armas de la dominación racista o neocolonial sobre un pueblo o un Estado". Todd S. Milliard, Overcoming Post-Colonial Myopia: a call to recognize and regulate Private Military Companies, 176 Military Law Review, 1-96 (2003).

47 República de Sudáfrica, op. cit.

48 Protocolo I adicional a los Convenios de Ginebra de 1949 relativo a la protección de las víctimas de los conflictos armados internacionales (1977).

49 au.int/AHG/49(IV), Resolución 49(IV) de la Organización para la Unidad Africana "sobre las actividades de los mercenarios".

50 Convención Internacional contra el reclutamiento, la utilización, la financiación y el entrenamiento de mercenarios, op. cit. 51 Íd.

52 Elke Krahmann, From 'Mercenaries' to 'Private Security Contractors': The (Re) Construction of Armed Security Providers in International Legal Discourse, 40 Millenium: Journal of international Studies, n. ${ }^{\circ}$ 2, 356 (2012).

53 Christopher Kinsey, Coporate soldiers and International Security, 5-6 (Routledge, 2006); Pilar Pozo Serrano \& Maria Lourdes Hernández Martín, El Marco Jurídico de las CMSP. Reflexiones a propósito de la experiencia en Irak, 23 Anuario Español de Derecho Internacional, 315-351 (2007).

54 Thomas K. Adams, Private Military Companies: Mercenaries of the 21st Century, 13 Small Wars and Insurgencies, n. 2, 54-67 (2002).

55 Clive Walker, \& Dave Whyte, Contracting out War? Private Military Companies, Law and Regulation in the United Kingdom, 54 The International and Comparative Law Quaterly, n. ${ }^{\circ}$ 3, 684 (2005).

56 Paul Jackson, 'War is much too serious a thing to be left to military men': Private military companies, combat and regulation, 5 Civil Wars, n. ${ }^{\circ}$, 30-55 (2002).

57 Milliard, op. cit., 7-8; Renée De Nevers, op. cit., 179; José L. Gómez del Prado, op. cit., 442.

58 Mohamad Ghazi Janaby, The Legal Status of Employees of Private Military/Security Companies Participating in U.N. Peacekeeping Operations, 13 Northwestern Journal of International Human Rights, n. ${ }^{\circ}$ 1, 101 (2015); Renée De Nevers, op. cit., 179; José L. Gómez del Prado, op. cit., 443.

59 José L. Gómez del Prado, op. cit., 442.

60 Ulrich Petersohn, Reframing the anti-mercenary norm: Private military and security companies and mercenarism, 69 International Journal, n. ${ }^{\circ}$, $476(2014)$.

61 Íd.

62 Thomas K. Adams, op. cit.

63 Ulrich Petersohn, op. cit., 480.

64 Shannon Bosch, op. cit., 41.

\section{Licencia Creative Commons CC BY 4.0}

Para citar este artículo/To cite this article: Mario Iván Urueña-Sánchez, El personal de las Compañías Militares y de Seguridad Privadas (CMSP): ¿Contratistas civiles o mercenarios?, 69 Vniversitas (2020). https://doi.o $\mathrm{rg} / / 10.11144 /$ Javeriana.vj69.epcm 